\title{
Study on Working Diagnosis of Rod Pumping Wells Based on Machine Learning
}

\author{
Peng Gao ${ }^{1, ~ *, ~ X u e f e n g ~ J i n ~}{ }^{1}$, Jieyu $\mathrm{Du}^{2}$, Yu Han ${ }^{2}$, Yanhui Zhu ${ }^{1}$ \\ ${ }^{1}$ Institute of Oil and Gas Recovery, Engineering Technology Research Institute of Huabei Oilfield Company, Renqiu, China \\ ${ }^{2}$ Institute of Engineering Technology, The Fourth Exploit Factory of Huabei Oilfield Company, Langfang, China
}

\section{Email address:}

cyy_gp@petrochina.com.cn (Peng Gao), cyy_jinxf@petrochina.com.cn (Xuefeng Jin), cyy_djy@petrochina.com.cn (Jieyu Du)

${ }^{*}$ Corresponding author

\section{To cite this article:}

Peng Gao, Xuefeng Jin, Jieyu Du, Yu Han, Yanhui Zhu. Study on Working Diagnosis of Rod Pumping Wells Based on Machine Learning. Science Discovery. Vol. 8, No. 1, 2020, pp. 7-11. doi: 10.11648/j.sd.20200801.13

Received: February 6, 2020; Accepted: April 3, 2020; Published: April 17, 2020

\begin{abstract}
The working condition diagnosis of pumping wells is an important basis for judging it's running condition. For a long time, indicator diagram is used to solve the wave equation by mathematical method to get the downhole pump indicator diagram which is obtained to judge and analyze the working condition of oil pumping equipment. In recent years, with the development of AI (artificial intelligence) technology, how to combine artificial intelligence technology with rod pumping wells lifting technology to provide a new way of thinking for pumping wells lifting and improve the intelligence level to achieve real-time control of the well has become a difficult problem in front of technicians. After several years of research, technicians in huabei oilfield innovatively proposed to establish working diagnosis model of pumping wells through machine learning neural network algorithm. The model can realize working diagnosis of oil well by using a large number of labeled indicator diagram and working diagnostic data as training samples. As long as the labeled data provided is of high accuracy and large amount, the accuracy of the model can reach more than $90 \%$. The model is simple in structure, fast in operation speed, and has high application value in the field. Several rod pumping wells were randomly selected from a working area of huabei oilfield, and the above model was used to diagnose the working conditions. The results showed that the average error between the diagnosis results and the real ones was only $7 \%$, less than $10 \%$ of the common requirements, which met the needs of engineering application.
\end{abstract}

Keywords: Machine Learning, Rod Pumped Well, Neural Network, Working Diagnostic

\section{基于机器学习的抽油机井工况诊断研究}

\author{
高鹏 $^{1^{*}}$, 金学锋 ${ }^{1}$, 杜婕妤 ${ }^{2}$, 韩羽 ${ }^{2}$, 朱延慧 ${ }^{1}$ \\ 1华北油田公司工程技术研究院采油采气所, 任丘, 中国 \\ 2华北油田公司第四采油厂工程技术研究所, 廊坊, 中国
}

邮箱

cyy_gp@petrochina.com.cn（高鹏），cyy_jinxf@petrochina.com.cn（金学锋），cyy_djy@petrochina.com.cn（杜婕妤）

摘要: 抽油机井功图工况诊断是判断抽油机运行状况的重要依据, 长期以来采用地面功图利用数学方法求解波动方程 得出井下泵功图来判断和分析抽油设备的工作状况，存在涉及参数众多和运算速度较慢的问题; 近年来随着AI（人工 智能）技术的发展，如何将人工智能技术和抽油机举升技术相结合，为抽油机举升提供全新的思路方案、提高智能化 水平达到对油井的实时控制成为摆在技术人员面前的一道难题。华北油田技术人员经过近几年研究，创新性地提出通 过机器学习神经网络算法建立抽油机井工况诊断模型, 采用大量带标签的功图和诊断结果数据作为训练样本对模型训 
练后可以实现对油井的智能诊断, 只要提供的标签数据准确度高、数据量大, 模型准确度可以达到 $90 \%$ 以；该模型 结构简单, 运算速度快, 在现场具有较高的应用价值。在华北油田某工区随机选取若干口抽油机井, 利用上述模型对 工况进行诊断, 结果表明, 诊断结果与实际结果的平均误差仅为 $7 \%$, 小于 $10 \%$ 的常用要求, 满足工程应用需要。

关键词: 机器学习, 抽油机井, 神经网络, 工况诊断

\section{1. 引言}

抽油机井功图工况诊断技术自上世纪 80 年代以来, 经 过技术人员几十年的努力已经较为成熟, 平均诊断误差在 $10 \%$ 以内，并已在全国各大油田推广应用[1-3]。但是，目 前利用数学方法求解波动方程得出井下泵功图进行工况 诊断的方法存在以下两个问题: 一是计算方法涉及到的参 数较多, 对参数的准确性要求较高, 如阻尼系数、液体粘 度、抽油杆直径、应力波传播速度、曲柄旋转角速度、傅 里叶系数及展开级数项等, 为了便于计算, 每个参数都选 取的是定值（实际中有的参数随井深发生变化, 如抽油杆 直径, 有的参数在单冲次内发生变化, 如抽油机电机变速 运行时每个时刻的曲柄旋转角速度都在发生变化）, 而每 个参数设置是否合理对诊断结果具有较大的影响, 这就要 求对每口井设置合理的计算参数; 二是计算过程迭代次数 多, 计算时间长; 求解波动方程要求从井口往下逐段进行 受力计算, 而每段计算时的傅里叶系数都要循环至展开级 数项的次数, 该次数选择过少导致计算精度变差, 选择过 多导致计算时间变长, 单井单功图计算时长约 5-10秒, 尤 其是针对大量油井数据同时计算时运算更慢, 无法实现油 井实时优化控制的需要。

近年来, $\mathrm{AI}$ 技术的发展为智能工况诊断提供了技术手 段, 利用该技术实现油井的智能工况诊断, 可以避免采用 数学方法解波动方程的复杂程序, 极大地提高运算效率, 为后续其他抽油机井计算、控制等智能程序的开发、嵌套、 运行提供便捷性，从而实现对油井“一个冲程控制一次、
一个冲程计算一次”的实时匹配油井生产状态的人工智能 采油精细控制模式，大大提高油井的智能化水平; 该技术 核心主要为机器学习、语言处理、传感器采集等方面, 通 过神经网络算法搭建油井工况诊断模型, 并收集前期油井 运行的功图数据和已知的诊断结果数据作为训练样本, 对 建立的模型进行训练, 得到具有对新数据进行预测功能的 工况诊断模型, 实现对油井的智能诊断。

\section{2. 抽油机井功图工况诊断方法}

抽油机井的主要设备抽油杆和抽油原一直在井下数 千米的环境下工作, 工况复杂恶劣, 发生故障的可能性较 高。在发生故障时, 如果不能及时诊断, 将会影响生产。

通常是利用井下泵功图进行诊断, 一种是采用设备测 量抽油泵的位移和载荷, 即井下潅示功图, 判断抽油机井 的工作状况。另一种就是通过地面功图计算出井下泵功图; 由于功图采集便捷可靠, 示功图诊断技术得到了国内外采 油工程师的青睐, 成为重要的研究方向。

\section{1. 采集井下泵示功图诊断法}

美国首先试验了采用井下动力仪直接测量井下示功 图。其优点在于可以除掉许多不确定因素对示功图造成的 影响, 如抽油杆的振动等 [1], 测量效果见图1。

测量出井下泵功图后, 对其进行特征参数提取、阈值 判断得出诊断结果。

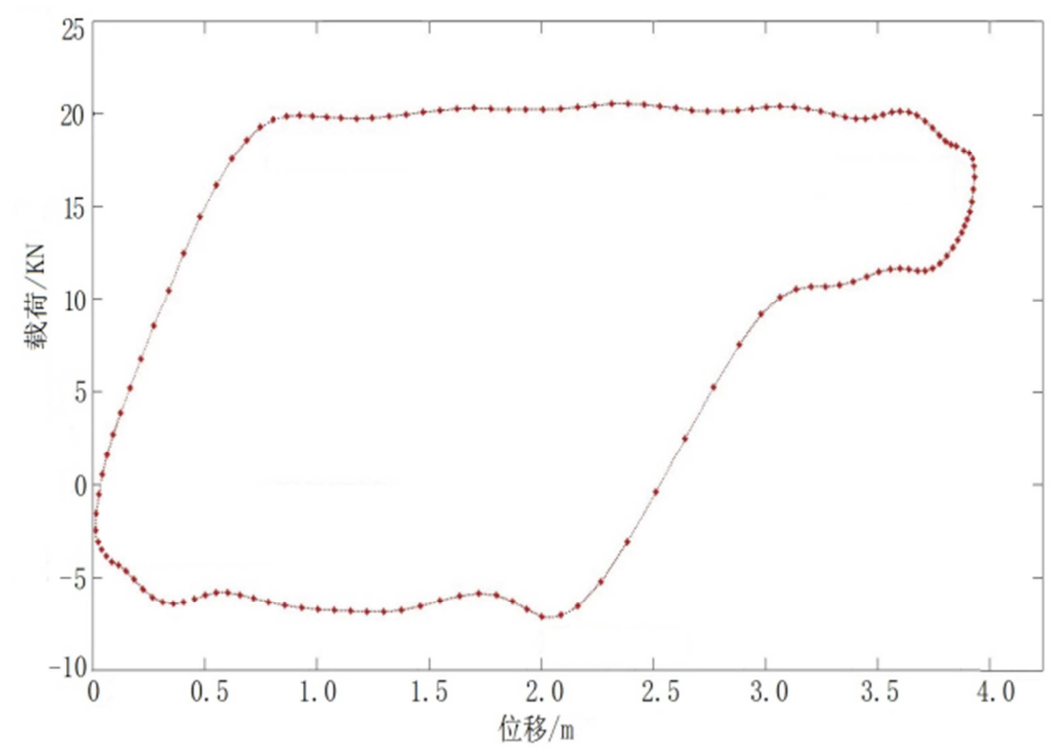

图1 测量的井下原功图。 
但该方法设备工艺复杂, 费用高, 而且井下设备的维 护艰难, 所以不符合实际需要, 难以得到推广。

\section{2. 求解洜示功图诊断法}

由于井下直接采集泵功图的设备应用困难, 所以人们 采用有杆抽油系统的力学模型分析, 由地面示功图经计算
机处理得到井下洜功图, 再依据其变化曲率得出凡尔开闭 点以及功图特征对油井进行诊断。该方法将理论研究与实 际情况相结合, 建立定向井的振动力学模型, 对百功图进 行模式识别, 实现有杆抽油系统的故障诊断[2], 见图2。

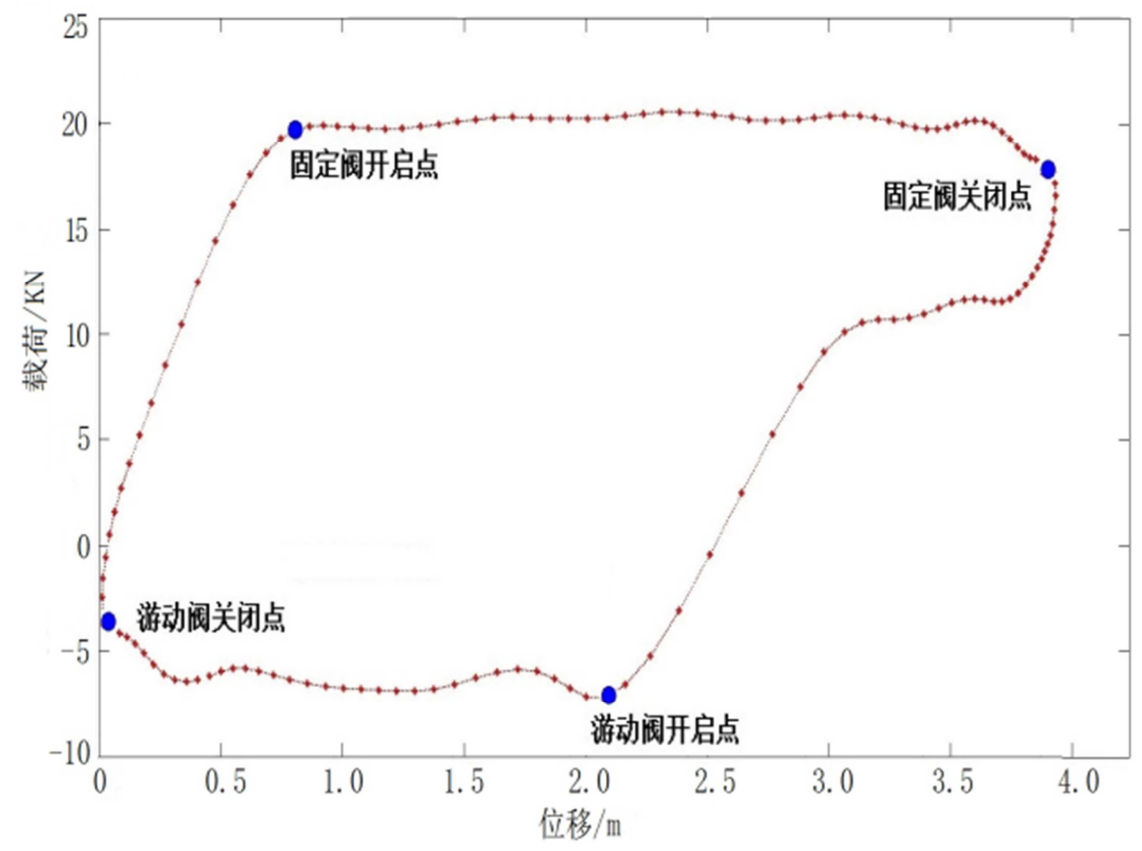

图2 曲率法确定原功图凡尔开闭点模型。

解波动方程的数学算法运算程序复杂、计算周期长、 运维成本高, 对于载荷波动频繁、生产不稳定的井确定凡 尔开闭点准确度也较低; 因此, 提出了基于机器学习算法 建立抽油机井工况诊断模型的新思路, 该诊断方法直接快 速, 不需要复杂运算, 具有运算简单快捷的优点。

\section{3. 基于机器学习的抽油机井功图工况诊断模型}

\section{1. 利用地面功图进行工况诊断存在的问题及解决方法}

由于目前的载荷位移传感器采用的是加速度感应 采集载荷位移数据, 这种采集模式存在一个问题: 光杆 运行加速度较小的地方, 载荷位移点分布较散, 光杆运 行加速度较大的地方, 载荷位移点分布十分密集 [4], 见 图3。尤其是抽油机井经智能控制变为悬点非正弦变速 运行时, 加速度、速度变化与传统悬点正弦运动规律完 全不符, 功图采集容易出现丢点现象, 使单冲次功图采 集的点数达不到 144 个点的模型输入要求, 而由于模型 输入数据必须要求所有功图为固定点数 (如 144 个点或 200 个点) 的数据模式, 这就要求对采集的功图数据进 行调整处理。

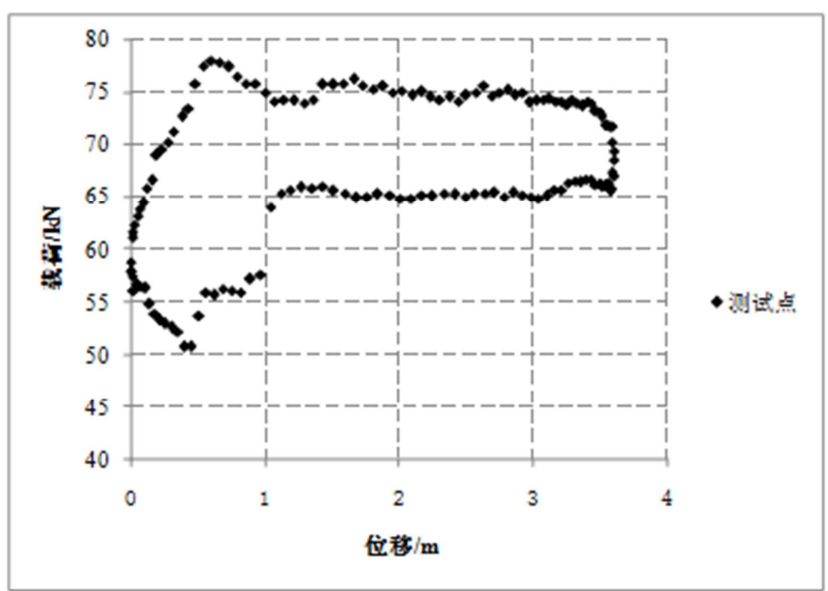

图3 某抽油机井某时刻载荷位移采集结果。

为了解决这一技术难题, 编写了一种功图修正算法。 利用该算法, 系统可以根据功图离散点的分布结构, 以第 一个点为起点, 之后按顺序自动将功图数据彼此相邻点按 照连续光滑的曲线连接起来, 形成闭合的初步图形, 从而 转化为功图图片格式, 再用一定的工具对图片标准化, 转 变为均匀像素点的矩阵, 每个功图矩阵都包含144个点或 200 个点, 存入系统numpy数组中, 作为模型输入并参与 模型建立与训练。利用功图修正算法对功图处理之后的效 果见图4。 


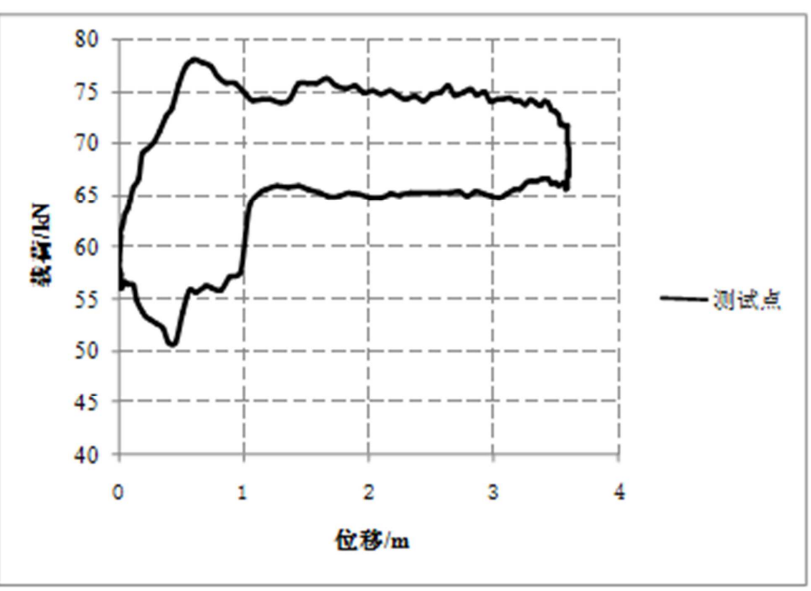

图4 光滑曲线连接后的功图。

从图4中可以看出, 利用功图修正算法修正之后的功 图, 在未改变功图形状的前提下, 增强了数据点分布的均 匀性, 满足了模型对功图数据输入为固定点数的要求。

\section{2. 基于机器学习的抽油机井功图工况诊断模型}

利用采集的地面功图, 转化为固定点数的功图矩阵 数据后, 就可以作为模型的输入数据。

模型采用ResNet_50(一种50层的卷积神经网络)的网 络结构, 由于功图转化为矩阵数据后维度较高(一个功图 200 个采集点对应 200 维矩阵数据), 该网络结构在提取高 维度特征向量的同时也可以保证模型的准确率以及计算 速度。一般认为神经网络的每一层分别对应于提取不同层 次的特征信息, 有低层、中层和高层之分, 而网络层数越 深，提取到的不同层次的信息就会越多，而不同层次间的 层次信息组合也就越多, 随着网络层数的增加会出现梯度 消失和收玫变慢的问题，通过优选选择ResNe_50t模型结 构很大程度上避免了此类问题, 能够更全面地提取示功图 的特征, 使诊断结果更加快速准确。

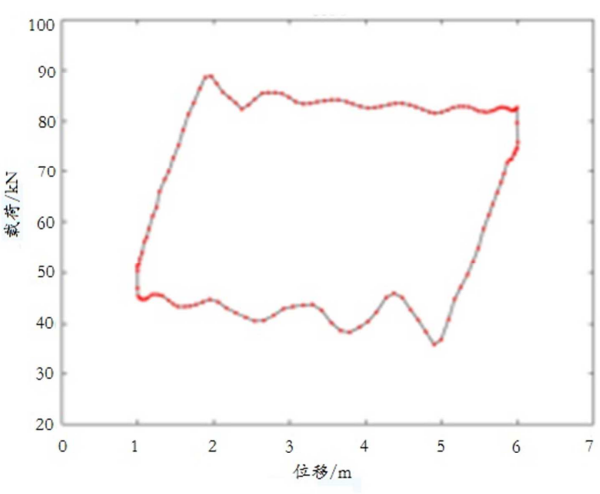

功图

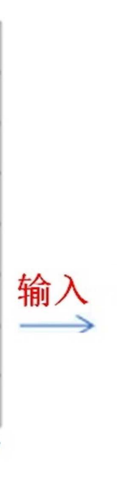

模型在训练过程中自动不断地调整神经网络节点参 数, 最终确定出一套适应于智能诊断的高准确率节点参数, 训练结束后, 整体模型的结构和节点参数值就保存在一个 ckpt文件中，将此模型植入抽油机井智能控制的软件系统 中, 工况诊断时可以直接调用训练好的模型对数据进行预 测。由于模型不需要对功图数据进行解波动方程的数学运 算程序, 仅需运用训练时已学习到的知识 (网络结构和节 点参数) 对数据进行认知预测, 因而模型运算时间大幅减 少, 经测试, 单井单功图运算时间在 5 毫秒以内, 为实现 油井数据的实时分析与控制打下了基础。

采用12种工况样本数据对模型进行训练和测试, 训练 集与测试集数据之比为 $4: 1$, 具体数据样数见表 1 。

表1 工况诊断模型训练与测试数据集。

\begin{tabular}{llll}
\hline \multirow{2}{*}{ 工况类别 } & 样本数据库 & & \\
\cline { 2 - 4 } & 训练数据数量 & 测试数据数量 & 训练标签 \\
\hline 连抽带喷 & 16633 & 554 & 1 \\
气锁或严重液击 & 18243 & 608 & 2 \\
\hline
\end{tabular}

\begin{tabular}{|c|c|c|c|}
\hline \multirow{2}{*}{ 工况类别 } & \multicolumn{3}{|l|}{ 样本数据库 } \\
\hline & 训练数据数量 & 测试数据数量 & 训练标签 \\
\hline 杆断或传感器失灵 & 12951 & 431 & 3 \\
\hline 杆在泵口断脱 & 12006 & 400 & 4 \\
\hline 气体影响 & 35593 & 1186 & 5 \\
\hline 凡尔漏失 & 22818 & 760 & 6 \\
\hline 供液不足游动凡尔漏失 & 31500 & 1050 & 7 \\
\hline 氺上挂 & 17085 & 570 & 8 \\
\hline 葲下碰 & 20686 & 690 & 9 \\
\hline 卡洜 & 11940 & 398 & 10 \\
\hline 洜工作正常 & 51653 & 1722 & 11 \\
\hline 供液不足 & 39628 & 1321 & 12 \\
\hline
\end{tabular}

\section{4. 现场应用}

基于java平台基础上利用该模型编制了抽油机井智能 管控系统, 对油井数据进行了实际测试, 测试效果见图6。 统计结果显示, 模型对测试数据的准确率 $93 \%$, 平均误差 $7 \%$, 符合现场应用需求。 
功图数据乎功图

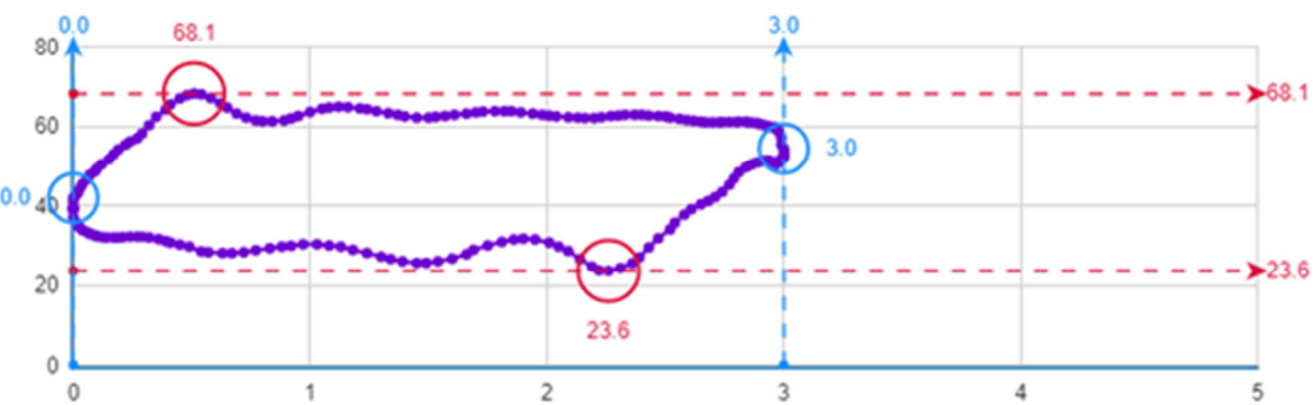

智能模块

产液量 $\quad 0=30 \quad 14.01 \quad$ 工况

图6 某井示功图诊断结果。

\section{5. 结论}

1) 针对悬点非正弦变速运行后井口示功仪采集数据易 丢点的问题, 通过将离散的功图数据转化为连续的图 形模式, 并从功图图形中提取出包含固定点数的均匀 像素点的矩阵, 解决了模型需要输入固定点数的数据 才能运行的难题。

2) 利用机器学习ResNet神经网络, 搭建了抽油机井工况 诊断模型, 该模型区别于常规的解波动方程的数学算 法模式, 运行速度达到毫秒级, 可以实现大量油井数 据的实时运算、诊断, 为人工智能技术在抽油机方面 的应用提供了借鉴。

3) 现场测试情况表明, 基于机器学习的功图工况诊断模 型平均相对误差仅为 $7 \%$, 小于 $10 \%$ 的常用要求, 满 足实际应用需求, 因此后续可以借鉴机器学习方法开 发功图算产模型等，实现油井“一个冲程控制一次、 一个冲程计算一次”的实时匹配油井生产状态的人工 智能采油精细控制模式, 大力推进油井的智能化水平。

\section{致谢}

本文为中国石油华北油田公司科研项目《机采井智能 采油技术研究与应用》的阶段性成果之一。

\section{参考文献}

[1] 张兴华, 王俊琦, 高志光, 郑欣. 计量油井产量的功图法技 术 $[J]$.河南石油, 2005, 19(5): 38-40。

[2] 唐庆, 王新红, 孙福山, 檀朝东, 关成尧.抽油机井功图法 量油技术先导试验 $[J]$.油气井测试, 2006, 15(6): 64-65。
[3] 严长亮, 彭勇.泵示功图单井自动量油技术研究[J].西安石油 大学学报(自然科学版)，2006, 21(6): 92-95。

[4] 高鹏, 常鹏刚, 张胜利, 金学锋, 户虎等. 基于地面功图功 图算产模型建立与应用 [J].钻采工艺，2018，41(2): 51-53。

[5] 韩赢, 张翼翔.浅谈斜井有杆葲葲况诊断[J].石化技术, 2018, 25(2): 186-187。

[6] 侯延涁, 陈炳均, 高宪文. 基于GM-ELM的有杆原抽油井故 障诊断 [J].东北大学学报(自然科学版)，2019, 40(12): 1673-1678。

[7] 袁文琪, 胡敏.基于示功图的油井故障诊断专家系统研究 [J]. 电子设计工程, 2015，23(18): 119-122。

[8] 陈德春, 陈逸芸, 孟红霞, 秦延才, 庄栋.助力深抽有杆葲 井工况诊断模型 $[\mathrm{J}]$.特种油气藏, 2015，22(03)：144-147。

[9] 饶建华, 刘宏昭, 李冬平, 黄伟, 漂长影. 定向井有杆抽油 系统动态数值仿真与预测 [J]. 石油机械, 2004, 32(8): 4-6。

[10] 薛国民, 沈毅.油井计量方法及关键技术发展方向 [J].工业计 量, 2006, 16(14): 14-16。

[11] 丁建林, 姜建胜, 刘廷等.抽油机变频调速智能控制技术研 究 [J].石油机械, 2003，31(1): 18-20。

[12] S. G. Gibbs. Computer Diagnosis of Down-hole Conditions in Sucker-Rod Pumping Wells [J]. Journal of Petroleum Technology, 1966, 18 (1): 27-29。

[13] S.G. Gibbs. A Method of Determining Sucker Rod Pump Performance [J]. U. S. Patent. 1967, 3 (3): 18-20。

[14] Dory, D. R and Schmidt Z. An Improved Model for Sucker Rod Pumping[ J]. SPE Engineers Journal, 1983, 2 (2): 33-40。

[15] R. R. Dickinson. The Use of Pattern Recognition Techniques in Analyzing Down Hole Dynamometer Cards [J]. A Thesis for the Degree of Master of science, 1987, 3 (6): 187-192。 